\title{
The Value of Distributed Energy Resources to the Grid: Introduction to the Concepts of Marginal Cost of Capacity and Locational Marginal Value
}

\author{
Richard Tabors \\ Tabors Caramanis Rudkevich, \\ Boston, MA \\ rtabors@tcr-us.com
}

\author{
Panagiotis Andrianesis \\ Michael Caramanis \\ Boston University, \\ Boston, MA \\ panosa@bu.edu \\ mcaraman@bu.edu
}

\author{
Ralph Masiello \\ Quanta Technology, LLC., \\ Raleigh, NC \\ rmasiello@quanta-technology.com
}

\begin{abstract}
Distributed Energy Resources (DERs) are argued to be a significant benefit to the electric utility grid. While DERs generate significant benefits to their owners and as well as society, the compensation and operating structure of the distribution system of most utilities is such that DERs result in minimal benefits to the distribution system. As we show, the benefits correctly attributed to the distribution company (the wires company) are a function of what service (real, reactive power) the DER is able to provide, when and where, and at what level of certainty the DER is able to provide the service. We introduce the concepts of Marginal Cost of Capacity (MCC) and Locational Marginal Value (LMV) in the calculation of the value of DERs to the distribution system.
\end{abstract}

\section{Introduction}

The wholesale bulk power (Generation and Transmission) market has seen the acceptance of the concept of power generation as a Non-Wires Alternative (NWA) to transmission take hold since the introduction of FERC 1000 [1]. FERC 1000 created a form of Integrated Resource Planning (IRP) that is intended to be market driven and decentralized in that third parties are able to compete to build transmission and to propose NWAs to relieve transmission congestion or to address specific transmission system needs identified by a TSO/RTO. At the wholesale level the NWAs have focused on demand response and on both fossil and renewable resources located at strategic positions within the transmission system.

The distribution sector has adopted the vocabulary of NWAs with vigor arguing that these alternatives provide a breadth of advantages to society through the provision of energy, reserves and other ancillary services including reactive power compensation, and, most importantly, an alternative or delay to expensive and lumpy capital investments.

This paper focuses on identifying the value of Distributed Energy Resources (DERs) to the owner operator of the distribution system. In the context of the market within which the distribution system operator sees a profit and loss, the value provided by the DERs must be measured in terms of achievable savings. These savings consist of avoided costs of operation, and, as we demonstrate, most significantly (even though less so than many have argued) of avoided capital investment.

We describe a framework and methodology for establishing the Value of DERs to the grid that addresses the challenges posed by the push for DERs as a NWA. The framework is grounded in engineering and economics, draws on parallels from the wholesale concepts of locational marginal pricing adapted (i) to the distribution network in the context of Distribution Locational Marginal Pricing (DLMP) [2] - [7] and (ii) for valuing infrastructure investment, and establishes two new economic concepts that are key parts of the methodology.

The first is the Marginal Cost of Capacity (MCC) which is the methodology for allocating the capital and other costs of traditional wires upgrades to locations on a distribution system across the hours of the year.

The second is the Locational Marginal Value (LMV) of DERs as a NWA that establishes the value of an increment of a generic DER to the grid at each location and at each point in time.

\section{Background}


Unlike at the wholesale level where market design and oversight of market decisions are the purview of the FERC, at the distribution or retail level the decision to allow market-based solutions (non-wires solutions) belongs to the state legislatures and public service commissions. FERC's current rulemaking on distributed storage and ongoing investigation into DERs in the markets make this clear - FERC believes it can establish the rules for having DERs participate in wholesale markets but that decisions about retail or distribution level markets, including DERs as nonwires assets, are squarely in the domain of state policy.

Multiple US states have begun to evaluate the concept of considering DERs as a NWA to traditional distribution system ("grid") investments. NY has established the "Reforming the Energy Vision" (NY REV), which has required the regulated utilities to propose tariff based compensation to DERs for the value they provide to the grid in terms of avoided cost [8]. The NY REV established a tariff based approach which is inherently less granular in location and time and which makes use of "generic" capacity costs on a utility basis as opposed to specific capacity upgrade costs on a per feeder basis.

California does not have a proposed rulemaking or tariff as of yet, but has an active working group (WG) the Locational Net Benefit Analysis (LNBA) WG looking at the value of DERs [9]. The California LNBA WG addressed a much larger problem and established a framework that attempts to encompass all benefits of DERs to society - including reduced energy costs, impacts on locational marginal pricing, reduced emissions, improved resiliency, etc., locationally. The published work did not address locational benefits on a distribution circuit but stopped at the wholesale station using LMPs as the basis. The California Public Utilities Commission (CPUC) review commented that locational value on the same granularity as PV hosting, for instance, was a critical gap. Subsequent unpublished work reportedly addressed the locational benefits of DERs on a circuit in terms of circuit Ampere capacity (ampacity), but did not include losses or voltage effects.

The Illinois Next Grid legislation has a goal of recognizing the Value of DERs to the Grid [10]. Next Grid proposes a structure in which DERs would be incented or compensated as a NWA through a form of a decentralized IRP process.

Motivations for pursuing DERs as NWA include (i) encouraging more distributed resources as a matter of policy due to the economic, environmental, and other benefits from renewable distributed generation, (ii) reducing long term investment in grid assets that are regulated assets, the majority of which are built for peak load conditions and are underutilized a high percentage of the time, and (iii) gaining more flexibility in investment decisions against an uncertain future need [11].

The first principle in determining the Value of DERs to the distribution grid in terms of avoided costs is that all DER value is locational. That is because all the avoided costs for capacity and voltage are investment costs in response to actual or forecast needs of the system, which are always based on specific grid issues in specific locations. Even if society were to establish that there is an additional value of having excess distribution capacity (for example, to prepare for a sudden need for electrification of building space conditioning or transportation) as insurance against sudden load growth, the value of DERs would still be locational.

Recognizing that the value of DERs is and will always be locational is critical. If, for instance, it were deemed important to invest in order to have all circuit maximum loadings not greater than $75 \%$ of capacity instead of $90 \%$ of capacity, circuits that were already loaded below $75 \%$ would see no value of DER in terms of increasing capacity. Circuits that were loaded at $90 \%$ would see a greater value than those loaded at $85 \%$ or $80 \%$ depending upon the avoided costs of providing the additional capacity circuit by circuit.

On a given circuit, the ability of DERs to avoid capacity or voltage costs by providing real or reactive power depends very much upon the DER location relative to the grid constraint to be addressed. For instance, if a new warehouse is planned in the last half mile of a two-mile long circuit such that the first mile of the circuit would then be overloaded - DER in the first half mile would only help resolve the loading issue in the first half mile; it would have no effect whatsoever on the loading issue in the second half mile.

For radial distribution circuits (the most common structure in most instances) DERs must be downstream of the location of a loading issue in order to help mitigate the problem. This is true in general. Figure 1 illustrates this concept through a simple example.

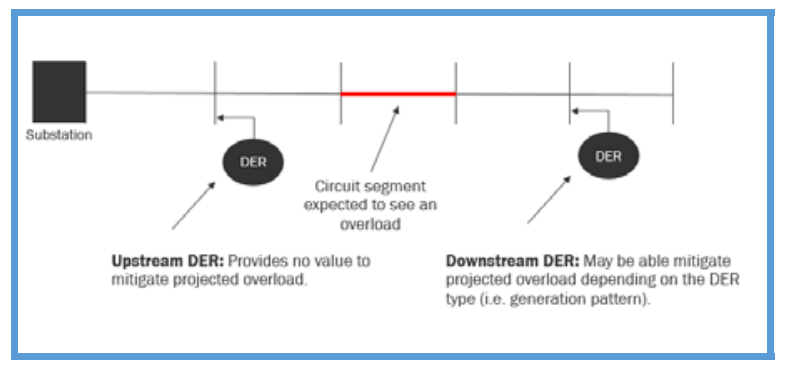

Figure 1. Illustration of DER overload mitigation 
Similarly, voltage problems on circuits are typically local in nature. Low voltage at the end of the circuit does not imply low voltage near or at the station. Indeed, attempting to cure the low voltage at one location by raising overall circuit voltages may cause too high a voltage in another location. Traditional mitigations to voltage issues are to deploy local apparatus that affects voltage locally, such as capacitors or voltage regulators. Controlling flicker is more difficult as capacitors and voltage regulators are not designed for rapid or frequent operation, so static VAR compensators or local DERs capable of varying real and reactive quickly are required, or alternatively the circuit must be re-engineered to decrease its impedance and thus the sensitivity of circuit voltages to changing PV output.

If we examine the hypothetical case where the first mile of a circuit needs to be reinforced and the argument that a generic DER only affects loadings on the upstream portions of the circuit, then this introduces the concept of how to place a locational value on the DER. If the DER midway on the overloaded portion of the circuit deferred the need to upgrade capacity on the first half of the circuit conceivably it could at maximum avoid half of the cost. The DER located at the downstream end of the affected portion of the circuit could, by contrast, avoid all the cost. So conceptually, the second DER is worth twice what the first DER is in terms of Value to the Grid.

This concept can be generalized. For any DER, its locational value is affected by the sensitivity of the circuit condition requiring capacity or voltage investments to the real and reactive power from that DER.

A third concept is important when we evaluate the Value to the Grid of specific DER technologies. Circuit capacity and voltage problems typically do not happen to the same extent across every hour of the year, and in fact may only occur for a limited number of hours a year. The DER technology must be able to provide real and reactive power when they are needed as well as where they are needed; thus, DER value is locational in time and in space.

In summary, there are three elements to valuing DERs. First is the avoided cost of required circuit upgrades and how that cost is apportioned among affected parts of the circuit. Second is how effective real and reactive power input from the DERs is at a given location of reducing or avoiding that cost. Third is how different DER technologies align with the temporal value of DERs and how much of the generic DER value a given technology can realize.

\section{Desired Features of DER Value to the Grid Methodology}

The methodology proposed for determining the DER value to the grid should be efficient, accurate, and fair. Efficiency denotes maximizing the flexibility of the system and further deferring large investments to accommodate for changes in load [12]. As for accuracy, DERs should be compensated for services they provide to the distribution grid by addressing different characteristics and capabilities of different DER technologies as well as addressing differences in locational and temporal value of DERs. An equitable and fair methodology limits impact to nonparticipating customers and avoids under and overcompensation. It further avoids double counting when some sources of DER value are compensated elsewhere, and supports the penetration of DERs that can provide value.

A simple connectivity analysis could be developed to assess the value of DERs for avoiding ampacity upgrades, if losses and other factors were ignored. However, we are concerned with capacity increases in a $10-20 \%$ range, never a doubling or more. Distribution losses are of the order of $6 \%$, spread among secondary transformers, high voltage conductors, and station transformers. Depending upon the DER location, it may garner capacity relief in addition to its own capacity by reducing losses and thus reducing demand upon upstream conductors and station transformers. Behind the meter DERs connected on the secondary would also relieve secondary transformer losses.

Additionally, circuit power factors vary widely by time of day and location. Typical circuit power factors might be in the range of $95 \%$. If DER VAR withdrawal and injection can be used to obtain near $100 \%$ power factor on a dynamic basis, then an additional $4-5 \%$ of ampacity can be freed up to deliver power. This is not insignificant.

Finally, managing circuit voltage levels is also important and a source of capacity upgrades. Some, such as adding capacitor banks, are fairly inexpensive. But if high voltages due to high PV penetration or high flicker levels are the issue, mitigation can be more expensive. DERs with smart inverters offer a lower cost approach, possibly. So the Value of DERs needs to consider controlled VAR injection and withdrawal.

It would be possible to develop approximations to each of these three issues - average loss factors can be used, average power factor improvement assumed, and a cost of traditional VAR management as with an SVC (Static VAR Compensator) used as a local proxy for the DER Value. But the three issues interact, and 
voltage / power factor issues are very locational. A framework rooted in full AC analysis that reflects the interaction of the multiple effects is ultimately a better approach.

There are two key concepts to explain before describing the methodology.

The first, the Marginal Cost of Capacity (MCC), is the allocation of specific traditional circuit upgrade costs to each section and node on the circuit pro rata the amount spent on each and the severity of the driving condition on each. For instance, if a circuit reconductoring is required on the first 10 sections of a circuit with 20 sections, the allocation of the reconductoring cost is determined by the relative length of each section and by the amount of overload on each section at each hour. The section closest to the substation will have the greatest overloads and the greatest MCC allocated.

The second, the Locational Marginal Value (LMV), is the incremental value of a $\mathrm{kW}$ or $\mathrm{kVAR}$ at each node in reducing the overloads, over or under voltages, etc., that cause the MCC. Thus each node has LMV assigned reflecting the value of an incremental $\mathrm{kW}$ or $\mathrm{kVAR}$ at that node in affecting all circuit conditions linked to an MCC.

Figure 2 illustrates an example of LMVs for both real (P-LMV) and reactive power (Q-LMV) in a congested feeder (congested lines are shown in red). LMVs increase as we move down along the feeder; the higher the congestion relieved upstream, the higher the value.

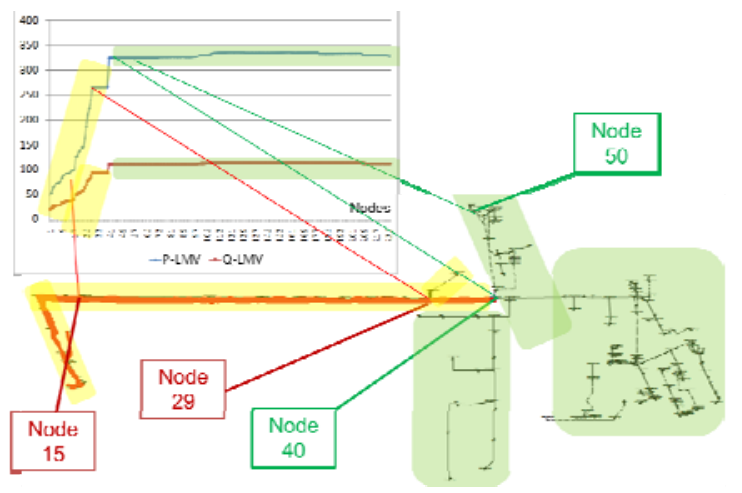

Figure 2. Illustration of LMVs in a congested feeder

\section{Methodology}

The proposed methodology consists of 3 steps. Step 1 is a preprocessing step, which calculates the constraint violation overload and the MCC. Step 2 is a pricing step, which derives the $\mathrm{kW}$ and $\mathrm{kVAR}$ LMVs for each time period and location. Step 3 is a procurement step, which derives the optimal addition of generic DERs required to relieve the overload.

For the purposes of this work, we use the branch flow model [13], [14] for a radial distribution network, which is presented in Appendix A. We note, however, that meshed networks are likely to appear more frequently in the future. Hence, we plan to extend our work to study meshed networks as well, based on approaches such as [15]. In the following subsections, we elaborate on each step of the methodology.

\subsection{Preprocessing}

The amount of overload is calculated for each hour of the anticipated yearly load profile. The (squared) magnitude of the current, $l_{i j, t}$, is derived by solving the branch flow model (listed in Appendix A). More specifically, ampacity constraint (A.6) is omitted and the following OPF problem is solved:

$$
\text { OPF-pre: (A.1), s.t. (A.2) - (A.5), }
$$

which is a Second Order Cone Programming (SOCP) problem because of (A.4).

Solving OPF-pre, the optimal values of $l_{i j, t}$ are derived and used to calculate the hourly overload $\Delta \hat{I}_{i j, t}=\left(\sqrt{l_{i j, t}}-I_{i j}^{\max }\right)^{+}$in Amps for each line segment $(i, j)$ exceeding its ampacity, where we define $(x)^{+}=\max \{0, x\}$.

The MCC is calculated from the best grid investment cost, $C$, which is obtained by a traditional wire solutions planning problem. We consider two cases.

In the first case, the project cost can be allocated to each line segment, e.g., a case of line upgrades. Let $c_{i j}$ be the cost for increasing line ampacity by $\Delta I_{i j}^{\max }$ (in Amps), with $\sum_{(i, j)} c_{i j}=C$, and $T_{i j}$ the number of hours the line upgrade is required within the year, i.e., the number of hours that the line is overloaded. We annualize $c_{i j}$ to equal its anticipated impact on the rate base; in this work, we scale by $a$. MCC overload penalty $w_{i j}$ is then defined as:

$$
w_{i j}=\frac{a \cdot c_{i j}}{\Delta I_{i j}^{\max } \cdot T_{i j}},
$$


where the MCC is measured in \$ per Amp of new capacity per (overloaded) hour, for a yearly period of one year.

In the second case, the investment cannot be allocated directly to the overloaded lines, e.g., the building of new lines as part of a reconfiguration scheme. Nevertheless, we can allocate the project cost to the overloaded lines, taking into account their maximum overload, $\Delta \hat{I}_{i j}^{\max }=\max _{t}\left\{\Delta \hat{I}_{i j, t}\right\}$, and length $L_{i j}$, as follows:

$$
c_{i j}=\frac{\Delta \hat{I}_{i j}^{\max } L_{i j} C}{\sum_{(i, j)} \Delta \hat{I}_{i j}^{\max } L_{i j}}
$$

MCC can be derived by (2) using the calculated value $\Delta \hat{I}_{i j}^{\max }$ instead of the actual increase in ampacity $\Delta I_{i j}^{\max }$ that results from the line upgrade.

\subsection{Pricing}

In this step we derive the DER LMVs. Penalizing the overload $\Delta I_{i j, t}$ by the MCC $\left(w_{i j}\right)$, the objective function becomes:

$$
\min _{P_{0}, Q_{0}, P_{i j}, Q_{i j}, v_{i}, l_{i j}, \Delta I_{i j}} c^{P} P_{0}+c^{Q} Q_{0}+\sum_{(i, j)} w_{i j} \Delta I_{i j}
$$

where we omitted the time index for brevity. $\Delta I_{i j}$ represents a new variable introduced for each overloaded line, so that the related penalties are only applied to $(i, j)$ with $\Delta I_{i j}>0$ during a specific hour.

Since the load flow solution is known from the previous step (we denote the known solution of the squared magnitude of the current by $l_{i j}^{0}$ ), we define the overload variable $\Delta I_{i j}$ using the $1^{\text {st }}$ order Taylor approximation as follows:

$$
\Delta I_{i j}=\frac{1}{2 \sqrt{l_{i j}^{0}}} l_{i j}+\frac{1}{2} \sqrt{l_{i j}^{0}}-I_{i j}^{\max }
$$

The penalty for the overload in the objective function represents the annualized pro-rated cost of the line, since we penalize only for the amount of new capacity needed in each hour, $\Delta I_{i j}$, instead of the maximum new capacity of the line $\left(\Delta I_{i j}^{\max }\right)$.

We can also consider alternative approaches, for instance, the Net Present Value of the annual revenue requirement of the capacity upgrade over an appropriate planning horizon. Our framework is applicable to alternative approaches, and in fact, the subject of policy choices. A key benefit of the marginal nature of our methodology is that the inclusion of the marginal avoided cost in the penalty $w_{i j}$ results in the DER investor and the customers sharing the avoided cost. If the entire avoided cost of planned traditional investments, including excess capacity, were included in the penalty, then all of the avoided cost could be captured by the generic DERs via the LMV mechanism. In such a case customers and ratepayers would realize no net savings.

For each hour in which overload was identified in the preprocessing step, the following optimization problem is solved:

$$
\text { OPF-pricing: (4), s.t. (A.2) - (A.5), and (5). }
$$

This is also an SOCP problem, and the LMVs are obtained as the shadow prices of constraints (A.2a) and (A.2b), i.e., $\lambda_{j}^{P}, \lambda_{j}^{Q}$. We refer to them as P-LMV and Q-LMV, respectively.

\subsection{Procurement}

In this step, we aim at deriving an optimal additional DER procurement that alleviates overload. Variables $P_{j, t}^{\mathrm{DER}} \geq 0$, and $Q_{j, t}^{\mathrm{DER}}$ represent real and reactive power procured from generic DERs at node $j$, time period $t$, at a cost equal to P-LMV and QLMV, respectively as estimated in the pricing step. The new objective function is defined by

$$
\min _{\substack{P_{0}, Q_{0}, P_{i j}, Q_{i j}, v_{i}, l_{i j}, P_{j}^{\mathrm{DER}}, Q_{j}^{\mathrm{DER}}}} c^{P} P_{0}+c^{Q} Q_{0}+\sum_{j \in N+}\left(\lambda_{j}^{P} P_{j}^{\mathrm{DER}}+\lambda_{j}^{Q} Q_{j}^{\mathrm{DER}}\right),
$$

where the time index $t$ is omitted for brevity. Note that $\lambda_{j}^{P}, \lambda_{j}^{Q}$ are parameters in this step, whose values are obtained from the previous step. Power balance constraints (A.2.a)-(A.2.b) are modified accordingly:

$P_{i j}-l_{i j} r_{i j}+P_{j}+P_{j}^{\mathrm{DER}}-\sum_{k: j \rightarrow k} P_{j k}=0, \quad \forall j \in N^{+}$, 


$$
Q_{i j}-l_{i j} x_{i j}+Q_{j}+Q_{j}^{\mathrm{DER}}-\sum_{k: j \rightarrow k} Q_{j k}=0, \quad \forall j \in N^{+} .
$$

Network constraints, such as service transformer rated capacities, may impose bounds on the real and reactive power quantities that can be procured by DERs at a certain node (and time period):

$$
\begin{gathered}
P_{j}^{\mathrm{DER}} \leq \bar{P}_{j}^{\mathrm{DER}}, \quad \forall j \in N^{+}, \\
-\bar{Q}_{j}^{\mathrm{DER}} \leq Q_{j}^{\mathrm{DER}} \leq \bar{Q}_{j}^{\mathrm{DER}}, \quad \forall j \in N^{+} .
\end{gathered}
$$

The optimal DER allocation is obtained by solving the following optimization problem, which is also an SOCP:

$$
\text { DER-opt: (7), s.t. (A.3) - (A.6), (8), and (9). }
$$

The solution of (10) provides an estimate of the DER quantities required to satisfy the ampacity constraints at a minimal procurement cost. In the absence of bound constraints (9), the solution of (10) is a lower bound on the DER procurement cost. Including constraints (9), we get a more realistic estimate of the DER procurement cost. An advantage of the proposed optimal DER procurement is that all network constraints are observed eliminating the potential of excessive DER additions at one or more locations introducing new problems in back flow, high voltage, etc.

\section{Conclusions}

A key objective of the proposed methodology has been to enable the planner to rely on information which is in its planning province. More specifically, the determination of the best grid investment alternative and its cost is within the utility's domain and expertise. For instance, the planner should not have to make assumptions about the costs of new DERs that hypothetically could be procured or incented to be developed, in order to relieve constraints and achieve feasibility.

In many cases the best wires alternative may be too "large" or too "lumpy" to be economic when DER investment alternatives are considered; this becomes an issue. In other words, if the full cost of a large investment justified by economies of scale and higher future capacity were to be used to derive the Value of DERs, then DERs would be overvalued by unjustifiably high overload penalties.

In our proposed methodology there are two distinct remedies. First, the cost of the investment is annualized, i.e., the wires investment cost is translated to its annual impact on the rate base. Second, its cost is pro-rated to the capacity that load growth indicates will be required during the next year or the relevant planning horizon.

Annualizing and pro-rating introduces the notion of the MCC. We use this notion in the valuation of generic DERs that are in fact invariant of actual DER costs and capabilities. Reliance on current or future DER capabilities and costs or uncertain forecasts of multiyear load growth that are particularly risky when location is considered as well.

The LMV provides a valuation of a "generic" DER at each node. As the LMV has a unique value by hour of the year, it provides a mechanism to further determine the value of a specific DER technology by comparing the profile of that technology against the profile of the LMV. Different technologies will "fit" the problem to varying degrees and have technology specific values. Some technologies may actually aggravate the problem (such as additional PV beyond hosting capacity) and would show negative values. QLMV, the value of kVAR, can take positive or negative values depending upon whether reactive power should be injected or withdrawn at a given location and hour.

\section{Appendix A: Branch Flow Model}

We consider graph $(N, E)$ representing a radial distribution network, where $N=\{0,1, \ldots, n\}$ is the set of nodes (node 0 is the root), $N^{+} \equiv N \backslash\{0\}$, and $E$ is the set of edges, denoted by pairs $(i, j)$, i.e., lines that connect nodes $i$ and $j$, ordered by the $j$-th node. The radial structure allows a unique path from root node 0 to node $j$, in which node $i$ is the node preceding $j$ in this path.

For each node $i \in N, V_{i}$ is the magnitude of the voltage, $v_{i} \equiv V_{i}^{2}$, and $V_{i}^{\min }\left(V_{i}^{\max }\right)$ is minimum (maximum) voltage limit. For each line $(i, j), r_{i j}$ is the resistance, $x_{i j}$ the reactance, $I_{i j}$ the magnitude of the current, with $l_{i j} \equiv I_{i j}^{2}, I_{i j}^{\max }$ the ampacity, and $P_{i j}$ and $Q_{i j}$ the sending-end real and reactive power flow, respectively. $P_{i}$ and $Q_{i}$ are the net real and reactive injections at node $i$. A positive (negative) value of $P_{i}$ refers to generation (consumption); similarly for the reactive power.

Figure A.1 shows a representation of a tree network. Note that $P_{0}=P_{01}$ and $Q_{0}=Q_{01}$, and hence variables 
$P_{0}, Q_{0}$, can be obtained from the balance in the root node.

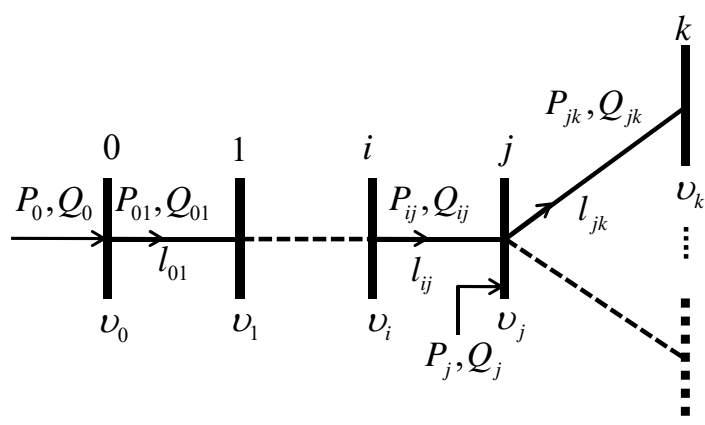

Figure 3. Tree network representation

The branch flow model is presented below. It is based on the DistFlow equations introduced in [13] and revised in [14]. This model simplifies conventional $\mathrm{AC}$ power flow equations for a radial distribution network. The formulation of our optimization problem representing an AC Optimal Power Flow (AC OPF) is listed below. The time index is omitted for brevity.

$$
\min _{P_{0}, Q_{0}, P_{i j}, Q_{i j}, v_{i}, l_{i j}} c^{P} P_{0}+c^{Q} Q_{0},
$$

subject to:

$$
\begin{gathered}
P_{i j}-l_{i j} r_{i j}+P_{j}-\sum_{k: j \rightarrow k} P_{j k}=0, \quad\left(\lambda_{j}^{P}\right) \forall j \in N^{+}, \\
Q_{i j}-l_{i j} x_{i j}+Q_{j}-\sum_{k: j \rightarrow k} Q_{j k}=0, \quad\left(\lambda_{j}^{Q}\right) \forall j \in N^{+},(\mathrm{A} \\
v_{j}=v_{i}-2\left(r_{i j} P_{i j}+x_{i j} Q_{i j}\right)+\left(r_{i j}^{2}+x_{i j}^{2}\right) l_{i j}, \quad \forall j \in N^{+}, \\
v_{i} l_{i j} \geq P_{i j}^{2}+Q_{i j}^{2}, \quad \forall(i, j) \in E \\
\left(V_{i}^{\min }\right)^{2} \leq v_{i} \leq\left(V_{i}^{\max }\right)^{2}, \quad \forall i \in N, \\
l_{i j} \leq\left(I_{i j}^{\max }\right)^{2}, \quad \forall(i, j) \in E,
\end{gathered}
$$

where $P_{i j}, Q_{i j} \in \mathfrak{R}$, and $v_{i}, l_{i j} \geq 0$.

The objective function (A.1) represents the cost of real and reactive power procured at the root node (i.e., at the T\&D interface), with $c^{P}$ the real power Locational Marginal Price (LMP), and $c^{Q}$ the reactive power compensation opportunity cost. Constraints (A.2a) - (A.2b) represent the real and reactive power balance at node $j$; their associated dual variables $\lambda_{j}^{P}, \lambda_{j}^{Q}$ denote the real and reactive power DLMPs at node $j$. Constraints (A.3) and (A.4) define nodal voltage and line current. Constraints (A.5) and (A.6) impose voltage and current limits.

Constraint (A.4) is a convex Second Order Cone Programming (SOCP) constraint. Note that in the DistFlow model, constraint (A.4) is an equality constraint, hence non-convex. However, for the cases of interest in this work, the relaxation is exact.

\section{Acknowledgements}

The work of P. Andrianesis and M. Caramanis was supported in part by the Sloan Foundation under Grant G-2017-9723 and NSF AitF grant 1733827.

\section{References}

[1] Federal Energy Regulatory Commission (FERC), 18 CFR Part 35, [Docket No. RM10-23-000; Order No. 1000] "Transmission Planning and Cost Allocation by Transmission Owning and Operating Public Utilities", Issued July 21, 2011.

[2] P. M. Sotkiewicz, and J. M. Vignolo, "Nodal pricing for distribution networks: efficient pricing for efficiency enhancing DG," IEEE Transactions on Power Systems, vol. 21, no. 2, pp. 1013-1014, 2006.

[3] N. G. Singhal, and K. W. Hedman, "An integrated transmission and distribution systems model with distribution-based LMP (DLMP) pricing," 2013 North American Power Symposium (NAPS).

[4] S. Huang, Q. Wu, S. S. Oren, R. Li, and Z. Liu, "Distribution locational marginal pricing through quadratic programming for congestion management in distribution networks," IEEE Transactions on Power Systems, vol. 30, no. 4, pp. 2170-2178, 2015.

[5] M. Caramanis, E. Ntakou, W. W. Hogan, A. Chakrabortty, and J. Schoene, "Co-optimization of power and reserves in dynamic T\&D power markets with nondispatchable renewable generation and distributed energy resources," Proceedings of the IEEE, vol. 104, no. 4, pp. 807 $-836,2016$.

[6] L. Bai, J. Wang, C. Wang, C. Chen, and F. Li, "Distribution locational marginal pricing (DLMP) for congestion management and voltage support," IEEE Transactions on Power Systems, vol. 33, no. 4, pp. 4061 4073, 2018.

[7] Z. Liu, Q. Wu, S. S. Oren, S. Huang, R. Li, and L. Cheng, "Distribution locational marginal pricing for optimal electric vehicle charging through chance constrained mixed- 
integer programming," IEEE Transactions on Smart Grid, vol. 9, no. 2 , pp. $644-654,2018$.

[8] State of NY Public Service Commission, Case 14-M0101 - Proceeding on motion of the Commission in regard to reforming the energy vision. Order establishing the benefit cost analysis framework. Jan 21, 2016.

[9] California Public Utilities Commission, Assigned Commissioner's ruling refining integration capacity and locational net benefit analysis methodologies and requirements; and (2) authorizing demonstration projects A and B, May 2016.

[10] Next Grid Illinois. https://nextgrid.illinois.gov

[11] Massachusetts Institute of Technology, The MIT Energy Initiative, "Utility of the Future," Cambridge, MA December 2016.
[12] P Andrianesis, M Caramanis, R Masiello, R Tabors, and S. Bahramirad. "Locational marginal value of distributed energy resources as non-wires alternatives", Draft working paper June 2018.

[13] M. E. Baran, and F. F. Wu, "Optimal capacitor placement on radial distribution systems," IEEE Transactions on Power Delivery, vol.4, no.1, pp. 725-734, 1989.

[14] M. Farivar, and S. Low, "Branch-flow model: relaxations and convexification - Part I," IEEE Transactions on Power Systems, vol. 28, no. 3, pp. 2554-2564, 2013.

[15] B. Kocuk, S. S. Dey, and X. A. Sun, "Strong SOCP relaxations for the optimal power flow problem," Operations Research, vol. 64, no. 6, pp. 1177 -1196, 2016. 